\title{
Validity of the limp model for porous materials: A criterion based on the Biot theory
}

\author{
Olivier Doutres, ${ }^{\text {a) }}$ Nicolas Dauchez, Jean-Michel Génevaux, and Olivier Dazel \\ Laboratoire d'Acoustique UMR CNRS 6613, Université du Maine, 72095 Le Mans Cedex 9, France
}

(Received 6 February 2007; revised 13 July 2007; accepted 13 July 2007)

\begin{abstract}
The validity of using the limp model for porous materials is addressed in this paper. The limp model is derived from the poroelastic Biot model assuming that the frame has no bulk stiffness. Being an equivalent fluid model accounting for the motion of the frame, it has fewer limitations than the usual equivalent fluid model assuming a rigid frame. A criterion is proposed to identify the porous materials for which the limp model can be used. It relies on a new parameter, the frame stiffness influence (FSI), based on porous material properties. The critical values of FSI under which the limp model can be used are determined using a one-dimensional analytical modeling for two boundary sets: absorption of a porous layer backed by a rigid wall and radiation of a vibrating plate covered by a porous layer. Compared with other criteria, the criterion associated with FSI provides information in a wider frequency range and can be used for configurations that include vibrating plates. (C) 2007 Acoustical Society of America. [DOI: 10.1121/1.2769824]
\end{abstract}

PACS number(s): 43.40.At, 43.20.Jr, 43.20.Gp, 43.50.Gf [KA] Pages: 2038-2048

\section{INTRODUCTION}

In recent years, there has been a strong effort for modeling the vibroacoustic response of multilayer systems containing porous materials. During the past decade, numerical methods such as finite element methods based on the Biot theory ${ }^{1,2}$ have been widely developed for automotive and aeronautic industries. Classical methods consider the displacements of the solid and fluid phases as variables, ${ }^{3,4}$ respectively $\mathbf{u}^{s}$ and $\mathbf{u}^{f}$, or a mixed formulation ${ }^{5,6}$ based on the displacement of the solid phase and the interstitial fluid pressure $p$. These numerical methods allow one to predict the structural and fluid couplings induced by the poroelastic medium without any kinematic or geometrical assumptions. However, for large size finite element models, these methods can require significant computational time.

To overcome this limitation, one can consider that the porous layer behaves like a dissipative fluid. Two porous "one-wave" formulations can be found: (i) the "rigid frame" model assumes that the solid phase remains motionless (Ref. 2, pp. 79-111) and (ii) the "limp" model assumes that the stiffness of the solid phase is zero but takes into account its inertial effects ${ }^{7-9}$ (Ref. 8, Chap. 5-1). Because the motion of the solid phase is considered in the limp model, this model has to be preferred for most of the applications as in transports, for example, car, train, aircraft, where the porous layers are bonded on vibrating plates. However, it is valid since the frame "flexibility" of the porous material has little influence on the vibroacoustic response of the system. The aim of this paper is to identify, for a given porous material, the frequency ranges for which the limp model can be used.

The approximation of the limp model was first proposed by Beranek ${ }^{7}$ and studied more in detail by Ingard. ${ }^{8}$ It was applied to the poroelastic formulation of Zwikker and

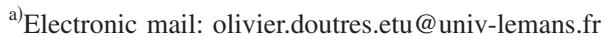

Kosten ${ }^{10}$ to model soft acoustical blankets in aircraft. More recently, Dazel et al. ${ }^{9}$ applied the limp approximation to an alternative displacement formulation based on Biot's poroelasticity equations. This simplified model accounts for (i) the dissipation mechanisms induced by the relative motion between the two phases and (ii) the effects of the motion of the solid phase using an inertia correction.

The validity of the limp model compared to the poroelastic model has been investigated by several authors. Beranek $^{7}$ proposed a simple criterion to identify the porous materials for which the limp assumption can be applied. This criterion is based on the ratio of the bulk modulus of the fluid phase $K_{f}$ over the bulk modulus of the solid phase $K_{s}$ : The limp assumption can be used if $K_{f} / K_{s}>20$. In his book, Ingard $^{8}$ investigates the absorption coefficient of a porous layer covered or not by a screen and backed by a rigid wall [as presented Fig. 1(a)]. He pointed out that the limp model is not valid in the low frequency range situated below the structural frame resonance of the porous layer because this region is mainly controlled by the stiffness. In the same way, Göransson ${ }^{11,12}$ investigated the validity of the limp model in the case of an aircraft double wall transmission problem and concluded that the limp model can be acceptable since no resonances of the frame are present in the frequency range of interest. The main conclusion of the previous works is that the use of the limp model depends not only on the properties of the porous layer but also on the boundary conditions which are applied to it.

In this paper, a criterion is proposed to identify the porous materials and the associated frequency ranges for which the limp model can be used according to the boundary conditions applied to the layer. The identification process relies on the derivation of a new frequency-dependent parameter, the frame stiffness influence (FSI), based on the properties of the porous material. This parameter is developed from the Biot theory ${ }^{1,2}$ and quantifies the intrinsic influence of the solid-borne wave $^{2}$ on the displacement of the interstitial 


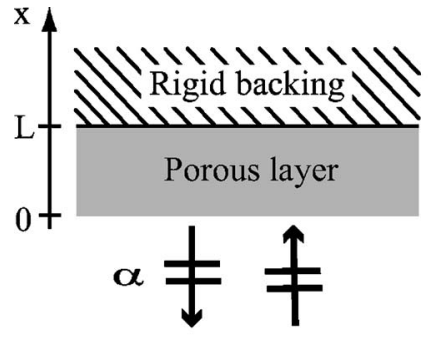

(a)

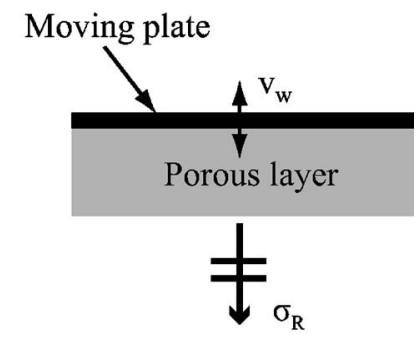

(b)
FIG. 1. Two one-dimensional configurations: (a) sound absorption of a porous layer backed by a rigid wall and (b) sound radiation of a porous layer backed by a vibrating wall.

fluid. Critical values of FSI above which the limp model cannot be used are determined for porous materials of thicknesses from 1 to $10 \mathrm{~cm}$ and for two boundary condition sets (see Fig. 1): (i) sound absorption of a porous layer backed by a rigid wall and (ii) sound radiation of a porous layer backed by a vibrating wall.

The first part of the paper presents the Biot and the limp models. The FSI parameter, which characterizes the influence of the frame, is then introduced and the method to determine the critical values of FSI is detailed. Critical values are given in Fig. 8 for two boundary condition sets and for ten porous thicknesses. The reader who is mainly interested in the application of the FSI criterion could go directly to the final part of the paper (Sec. VI). In this section, a short explanation on how to use the FSI criterion is proposed and it is illustrated with examples. Finally, a comparison with other criteria validates its efficiency.

\section{POROUS MATERIAL MODELING}

The limp model introduced in this paper is derived from the Biot theory adapted by Johnson, ${ }^{13}$ Allard, ${ }^{2}$ and Champoux. ${ }^{14}$ This poroelastic model accounts for frame motion, viscous and thermal dissipation mechanisms and is called the Biot model in this paper. In order to achieve a simple analytical description, the modeling is one dimensional, i.e., the porous layer has infinite lateral dimensions in the $y$ and $z$ directions, and only plane waves propagate in the $x$ direction (see Fig. 2). In this case, only two compressional waves are considered.

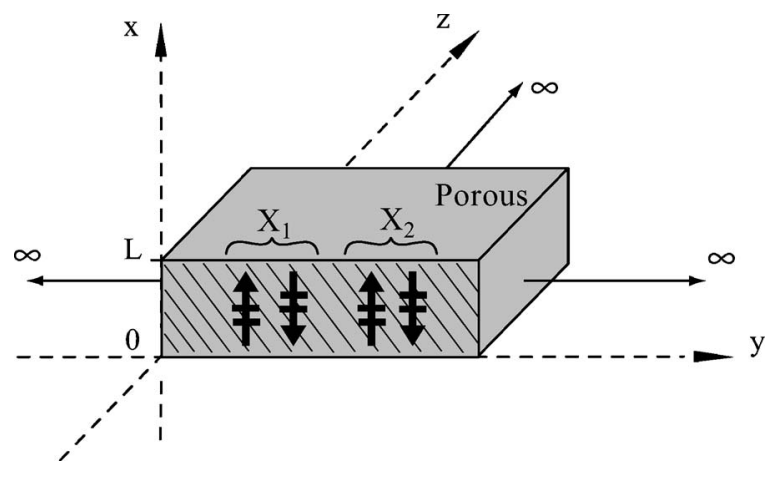

FIG. 2. One-dimensional porous modeling.

\section{A. Biot theory}

The motion of the poroelastic medium is described by the macroscopic displacement of solid and fluid phases represented by the vectors $\mathbf{u}^{s}$ and $\mathbf{u}^{f}$. Assuming a harmonic time dependence $e^{j \omega t}$, the equation of motion can be written in the following form: ${ }^{2}$

$$
\begin{aligned}
-\omega^{2} \widetilde{\rho}_{11} \mathbf{u}^{s}-\omega^{2} \widetilde{\rho}_{12} \mathbf{u}^{f}= & N \nabla^{2} \mathbf{u}^{s}+(\widetilde{P}-N) \boldsymbol{\nabla} \boldsymbol{\nabla} \cdot \mathbf{u}^{s} \\
& +\widetilde{Q} \nabla \nabla \cdot \mathbf{u}^{f}, \\
-\omega^{2} \widetilde{\rho}_{12} \mathbf{u}^{s}-\omega^{2} \widetilde{\rho}_{22} \mathbf{u}^{f}= & \tilde{Q} \nabla \nabla \cdot \mathbf{u}^{s}+\widetilde{R} \nabla \nabla \cdot \mathbf{u}^{f},
\end{aligned}
$$

where the tilde indicates that the associated physical property is complex and frequency dependent. The inertial coefficients $\widetilde{\rho}_{11}, \widetilde{\rho}_{22}$ are the modified Biot's density of the solid and fluid phases, respectively. The inertial coefficient $\widetilde{\rho}_{12}$ accounts for the interaction between inertial forces of the solid and fluid phases together with viscous dissipation. The modified Biot's density can be written in the following form:

$$
\begin{aligned}
& \tilde{\rho}_{12}=\rho_{12}+j \tilde{b} / \omega, \\
& \tilde{\rho}_{11}=\rho_{11}-j \tilde{b} / \omega, \\
& \tilde{\rho}_{22}=\rho_{22}-j \tilde{b} / \omega,
\end{aligned}
$$

with $\rho_{12}=-\phi \rho_{f}\left(\alpha_{\infty}-1\right), \rho_{11}=\rho_{1}-\rho_{12}$, and $\rho_{22}=\phi \rho_{f}-\rho_{12} \cdot \rho_{12}$ is the added mass due to tortuosity $\alpha_{\infty}, \rho_{1}$ the mass density of the porous material, $\rho_{f}$ the mass density of the interstitial fluid, and $\phi$ the porosity of the poroelastic material. The coefficient $\widetilde{b}$ is related to the viscous and inertial coupling between the two phases. This parameter was first assumed real and constant by Biot. ${ }^{1}$ Johnson et al. ${ }^{13}$ improved the model by introducing a frequency-dependent complex amplitude to account for the viscous effects. In the present work it is given by

$$
\begin{aligned}
& \tilde{b}=\phi^{2} \sigma \sqrt{1+j \frac{M}{2} \frac{\alpha_{\infty} \rho_{f}}{\phi \sigma} \omega,} \\
& M=\frac{8 \alpha_{\infty} \mu^{\prime}}{\sigma \phi \Lambda^{2}},
\end{aligned}
$$

where $M$ is the shape factor, $\mu^{\prime}$ is the viscosity of air, $\sigma$ the resistivity of the porous material, and $\Lambda$ the characteristic viscous length.

In Eq. (1) and (2), $\widetilde{P}$ and $\widetilde{R}$ are the bulk modulus of the solid and fluid phases, respectively, and $\widetilde{Q}$ quantifies the potential coupling between the two phases. For the majority of sound absorbing materials, these coefficients are ${ }^{2}$

$$
\begin{aligned}
& \tilde{Q}=(1-\phi) \tilde{K}_{f}, \\
& \tilde{R}=\phi \tilde{K}_{f},
\end{aligned}
$$




$$
\widetilde{P}=2 N^{*} \frac{1-\nu}{1-2 \nu}+\frac{\widetilde{Q}^{2}}{\widetilde{R}}=\hat{P}+\frac{\widetilde{Q}^{2}}{\widetilde{R}} .
$$

The effective bulk modulus of the air in the pores $\widetilde{K}_{f}$ is related to the thermal coupling between the two phases. In the initial paper of Biot, ${ }^{1}$ this parameter was real and constant. Champoux and Allard ${ }^{14}$ improved the model to account for the thermal effects:

$$
\tilde{K}_{f}=K_{a} / \widetilde{\beta},
$$

with $K_{a}$ the adiabatic incompressibility of air and

$$
\begin{aligned}
& \tilde{\beta}=\gamma-\frac{\gamma-1}{1+\left(j \omega \frac{k_{0}^{\prime} \rho_{f} \mathrm{Pr}}{\phi \mu^{\prime}}\right)^{-1} \tilde{G}^{\prime}}, \\
& \tilde{G}^{\prime}=\sqrt{1+j \frac{M^{\prime}}{2} \frac{k_{0}^{\prime} \rho_{f} \mathrm{Pr}}{\phi \mu^{\prime}} \omega,} \\
& M^{\prime}=\frac{8 k_{0}^{\prime}}{\phi \Lambda^{\prime 2}} .
\end{aligned}
$$

In Eqs. (11)-(14), $\gamma$ is the ratio of the specific heats, Pr the Prandtl number, $k_{0}^{\prime}$ the thermal permeability, and $\Lambda^{\prime}$ the thermal characteristic length. According to Eq. (11), the bulk modulus of the interstitial fluid varies from its isotherm value at low frequencies $\left(K_{f}=P_{0}=101.3 \mathrm{kPa}\right.$, the atmospheric pressure at $20{ }^{\circ} \mathrm{C}$ ) to its adiabatic value at high frequencies $\left(K_{f}=K_{a}=\gamma P_{0}=141.2 \mathrm{kPa}\right.$ at $\left.20^{\circ} \mathrm{C}\right)$.

$N^{*}$ and $\nu$ in Eq. (10) are the complex shear modulus and the Poisson ratio of the frame, considered as an isotropic material. $N^{*}$ corresponds to the second Lame coefficient $\mu$ and can be expressed in terms of the complex Young modulus in vacuo $E^{*}$ :

$$
N^{*}=\frac{E^{*}}{2(1+\nu)}=\frac{E(1+j \eta)}{2(1+\nu)},
$$

with $\eta$ the loss factor of the frame. Note that $N^{*}$ and $E^{*}$ are complex and frequency dependent. For the sake of simplicity, these coefficients are assumed to be constant in this work.

Since the model is one dimensional, the displacements are scalars and Eqs. (1) and (2) are rewritten as

$$
\begin{aligned}
& -\omega^{2} \widetilde{\rho}_{11} u^{s}-\omega^{2} \widetilde{\rho}_{12} u^{f}=\widetilde{P} \nabla^{2} u^{s}+\widetilde{Q} \nabla^{2} u^{f}, \\
& -\omega^{2} \widetilde{\rho}_{12} u^{s}-\omega^{2} \widetilde{\rho}_{22} u^{f}=\widetilde{Q} \nabla^{2} u^{s}+\widetilde{R} \nabla^{2} u^{f} .
\end{aligned}
$$

Equation (16) can be expressed in a more convenient way to identify the influence of the mechanical properties by introducing the bulk modulus of the solid phase in vacuo $\hat{P}$ [see Eq. (10)]. By solving Eq. (16) $-\tilde{Q} / \tilde{R} \times$ Eq. (17), one gets

$$
-\omega^{2} \frac{\widetilde{\rho}_{12}}{\phi} \widetilde{\Gamma} u^{s}-\omega^{2} \frac{\widetilde{\rho}_{22}}{\phi} \widetilde{\gamma} u^{f}=\hat{P} \nabla^{2} u^{s},
$$

with

$$
\widetilde{\Gamma}=\phi\left(\frac{\tilde{\rho}_{11}}{\tilde{\rho}_{12}}-\frac{\tilde{Q}}{\widetilde{R}}\right), \quad \tilde{\gamma}=\phi\left(\frac{\tilde{\rho}_{12}}{\tilde{\rho}_{22}}-\frac{\tilde{Q}}{\widetilde{R}}\right) .
$$

$\tilde{\gamma}$ is a notation introduced by Atalla et al. ${ }^{5}$ in the mixed displacement-pressure formulation. According to the Biot theory, the two poroelastic equations, Eqs. (17) and (18), can be solved to determine the propagation constants of the two compressional waves. A derivation of the complete system is proposed in Sec. II B.

\section{B. Poroelastic model: A two wave formalism}

Compared to classical Biot equations, ${ }^{1,2}$ the equations of motion proposed in this paper [Eqs. (17) and (18)] involve the bulk modulus of the frame in vacuo $\hat{P}$. The aim of this section is to derive an alternative and a more useful expression of the physical parameters of the Biot waves. This will be of interest for obtaining the FSI.

Using the vector $[\mathbf{w}]=\left[u^{s} u^{f}\right]^{T}$, Eqs. (17) and (18) can be reformulated as

$$
-\omega^{2}[\boldsymbol{\rho}][\mathbf{w}]=[\mathbf{M}] \nabla^{2}[\mathbf{w}],
$$

with

$$
[\boldsymbol{\rho}]=\left[\begin{array}{cc}
\widetilde{\rho}_{12} \widetilde{\Gamma} / \phi & \tilde{\rho}_{22} \tilde{\gamma} / \phi \\
\widetilde{\rho}_{12} & \widetilde{\rho}_{22}
\end{array}\right], \quad[\mathbf{M}]=\left[\begin{array}{cc}
\hat{P} & 0 \\
\widetilde{Q} & \tilde{R}
\end{array}\right] .
$$

Solution of the eigenvalue problem, $-\delta_{i}^{2}\left[\mathbf{w}_{i}\right]=\nabla^{2}\left[\mathbf{w}_{i}\right]$, gives the squared complex wave numbers $\delta_{i}^{2}$ of the two compressional waves $(i=1,2)$,

$$
\delta_{i}^{2}=\frac{1}{2}\left(\mathbf{A} \delta_{c}^{2}+\delta_{f}^{2}\right)+\frac{(-1)^{i}}{2} \sqrt{\left(\mathbf{A} \delta_{c}^{2}+\delta_{f}^{2}\right)^{2}-4 \mathbf{B} \delta_{c}^{2} \delta_{f}^{2}},
$$

where

$$
\mathbf{A}=\left(1-\frac{\tilde{Q} \widetilde{\rho}_{22} \tilde{\gamma}}{\widetilde{R} \widetilde{\rho}_{12} \tilde{\Gamma}}\right), \quad \text { and } \mathbf{B}=\left(1-\frac{\tilde{\gamma}}{\widetilde{\Gamma}}\right) .
$$

The wave numbers of the compressional Biot waves $\delta_{i}$ are written in terms of two characteristic wave numbers: $\delta_{f}$, the wave number of the rigid frame model described in Sec. II C [see Eq. (30)], and $\delta_{c}$, the wave number of a wave propagating in a medium that has the bulk modulus of the frame in vacuum and the density of the frame in fluid (it takes into account the inertial and viscous couplings):

$$
\delta_{c}=\omega \sqrt{\frac{\tilde{\rho}_{12} \widetilde{\Gamma}}{\phi \hat{P}}}=\omega \sqrt{\frac{\tilde{\rho}_{c}}{\hat{P}}},
$$

with

$$
\tilde{\rho}_{c}=\rho_{1}-\tilde{\rho}_{12} / \phi .
$$

Note that the expressions of the wave numbers $\delta_{i}$ [Eq. (22)] are equivalent to the classical expressions that can be found in Ref. 2 (p. 130).

The two eigenvectors can be written $\left[u_{1}\right]=\left[u_{1}^{s} u_{1}^{f}\right]^{T},\left[u_{2}\right]$ $=\left[u_{2}^{s} u_{2}^{f}\right]^{T}$. Each component of the vectors $\left[u_{i}\right](i=1,2)$, corresponds to the displacement of the solid phase $u_{i}^{s}$ and the fluid phase $u_{i}^{f}$ induced by the propagation of the Biot wave $i$. 
Using Eq. (18), the ratio of the displacement of the frame over the displacement of the air for the two compressional waves is given by

$$
\mu_{i}=\frac{u_{i}^{f}}{u_{i}^{s}}=\frac{\widetilde{\rho}_{12} \widetilde{\Gamma}}{\widetilde{\rho}_{22} \widetilde{\gamma}}\left(\frac{\delta_{i}^{2}}{\delta_{c}^{2}}-1\right) .
$$

This ratio indicates in which medium the waves mainly propagate. If it is less than 1, the wave is mainly supported by the frame and is referred to as the "frameborne wave." On the contrary, if the ratio is much greater than 1 , the corresponding wave is mainly supported by the saturating fluid and is referred to as the "airborne wave." By considering the whole set of porous materials used in this study and presented in Sec. III, one notices that the subscript $i=1$ corresponds to the airborne wave and the subscript $i=2$ corresponds to the frameborne wave.

In the considered geometry (see Fig. 2), the displacement of each phase is due to the propagation of two compressional waves traveling in both directions, and can be written in the following form:

$$
\begin{aligned}
& u^{s}(x)=X_{1}+X_{2}, \\
& u^{f}(x)=\mu_{1} X_{1}+\mu_{2} X_{2},
\end{aligned}
$$

where $X_{i}=S_{i} \cos \left(\delta_{i} x\right)+D_{i} \sin \left(\delta_{i} x\right)$ is the contribution of each compressional wave $i=1,2, S_{i}$ and $D_{i}$ being set by the boundary conditions.

\section{Equivalent fluid models: A one wave formalism}

Here, after a brief description of the classical rigid frame model, the limp model is presented. Both models are derived from the Biot theory presented in Sec. II B.

The rigid frame model assumes that the displacement $u^{s}$ is zero. This assumption applied in Eq. (17) gives a propagation equation on $u^{f}$,

$$
\tilde{K}_{f} \nabla^{2} u^{f}+\omega^{2} \frac{\tilde{\rho}_{22}}{\phi} u^{f}=0 .
$$

The compressional wave, solution of the propagation equation, is characterized by the wave number $\delta_{f}$,

$$
\delta_{f}=\omega \sqrt{\frac{\tilde{\rho}_{f}}{\tilde{K}_{f}}},
$$

where $\tilde{\rho}_{f}=\widetilde{\rho}_{22} / \phi$.

The limp model is based on the assumption that the frame has no bulk stiffness: $\hat{P}=0$. It is likely associated with "soft" materials like cotton and glass wool. Hence, by considering this assumption in Eq. (18), one gets a simple relation between the displacements of both solid and fluid phases:

$$
u^{s}=-\frac{\widetilde{\rho}_{22}}{\widetilde{\rho}_{12}} \frac{\widetilde{\gamma}}{\widetilde{\Gamma}} u^{f} .
$$

Then, substituting the solid displacement in Eq. (17) by Eq. (31) gives the propagation equation on $u^{f}$,

$$
\widetilde{K}_{f} \nabla^{2} u^{f}+\omega^{2} \widetilde{\rho}_{\text {limp }} u^{f}=0,
$$

where

$$
\tilde{\rho}_{\text {limp }}=\frac{\mathbf{B}}{\mathbf{A}} \widetilde{\rho}_{f},
$$

with $\mathbf{A}$ and $\mathbf{B}$ the two coefficients defined in Eq. (23).

The compressional wave, solution of the propagation equation, is characterized by the wave number $\delta_{\text {limp }}$ $=\omega \sqrt{\tilde{\rho}_{\text {limp }} / \widetilde{K}_{f}}$. According to the expression of the density $\tilde{\rho}_{\text {limp }}$, the wave number of the limp model can be expressed in terms of the wave number of the rigid frame model as

$$
\delta_{\operatorname{limp}}^{2}=\frac{\mathbf{B}}{\mathbf{A}} \delta_{f}^{2} .
$$

As in the rigid frame model, the bulk modulus of the limp model is the modified bulk modulus of the saturating fluid $\widetilde{K}_{f}$. However, the limp model takes into account the effect of the solid phase displacement by its modified effective density $\tilde{\rho}_{\text {limp }}$, which transcribes the inertia of the solid phase and its interaction with the fluid phase. This property enables use of the limp model for porous material mounted on a vibrating structure as long as the frameborne wave has no influence on the vibroacoustic behavior of the system. In this way, it is less restrictive than the rigid frame model.

\section{FRAME STIFFNESS INFLUENCE}

The aim of the following is to propose a parameter based on the properties of the porous material that quantifies the influence of the frame stiffness on the porous behavior. This parameter is called FSI.

\section{A. FSI development}

The use of the limp model is possible when the contribution of the frameborne wave is negligible in the considered application. This approximation implies the following in the expressions of the solid and fluid displacements [Eqs. (27) and (28)]:

(i) The contribution of the airborne wave $X_{1}$ is great compared to the contribution of the frameborne wave $\mathrm{X}_{2}$; this condition depends mainly on the boundary conditions: Two configurations will be studied in Sec. IV to set critical values of the FSI parameter.

(ii) Considering the fluid motion [Eq. (28)], the displacement ratio $\mu_{1}$ associated with the airborne wave is great compared to the displacement ratio $\mu_{2}$ associated with the frameborne wave: $\mu_{2} / \mu_{1} \ll 1$; this condition is independent from the boundary conditions and will be used to build the FSI parameter.

According to Eq. (26), the ratio $\mu_{2} / \mu_{1}$ can be written in terms of the wave numbers of the Biot waves $\delta_{i}$ [Eq. (22)] and the characteristic wave propagating mostly in the frame $\delta_{c}$ [Eq. (24)]:

$$
\frac{\mu_{2}}{\mu_{1}}=\frac{\left(\delta_{2}^{2} / \delta_{c}^{2}\right)-1}{\left(\delta_{1}^{2} / \delta_{c}^{2}\right)-1} .
$$


From the study of the behavior of 259 porous materials presented in Sec. IV B, the condition $\mu_{2} / \mu_{1} \ll 1$ is met when the following conditions are observed:

(i) $\quad r_{1}=\delta_{1}^{2} / \delta_{c}^{2}$ tends to 0 : This occurs when the wave number $\delta_{1}$ of the airborne wave is small compared to the wave number $\delta_{c}$.

(ii) $\quad r_{2}=\delta_{2}^{2} / \delta_{c}^{2}$ tends to 1 : This occurs when the wave number $\delta_{2}$ of the frameborne wave is close to the wave number $\delta_{c}$.

Both $r_{1}$ and $r_{2}$ ratios exhibit an asymptotic behavior as the frame stiffness becomes smaller: Their convergence is controlled by a common parameter presented in Sec. III B.

\section{B. A simplified parameter}

Let us first consider the asymptotic behavior of the ratio $r_{1}$. Substituting $\delta_{1}$ in the expression of $r_{1}$ for Eq. (22) gives

$$
r_{1}=\frac{\delta_{1}^{2}}{\delta_{c}^{2}}=\frac{1}{2}\left(\delta_{f}^{2} / \delta_{c}^{2}+\mathbf{A}\right)[1-\sqrt{1-\varepsilon}]
$$

with

$$
\varepsilon=\frac{4 \mathbf{B} \delta_{f}^{2} / \delta_{c}^{2}}{\left(\delta_{f}^{2} / \delta_{c}^{2}+\mathbf{A}\right)^{2}} .
$$

This expression shows that $r_{1}$ may tend to zero when $\varepsilon$ tends to zero. Hence, by using the first-order Taylor series expansion of the square root function $\sqrt{1-\varepsilon}=1-\varepsilon / 2$, it becomes

$$
r_{1}=\frac{\left(\mathbf{B} \delta_{f}^{2}\right) /\left(\mathbf{A} \delta_{c}^{2}\right)}{1+\delta_{f}^{2} /\left(\mathbf{A} \delta_{c}^{2}\right)} .
$$

By introducing the wave number of the limp model using Eq. (34), one gets

$$
r_{1}=\frac{\delta_{\text {limp }}^{2} / \delta_{c}^{2}}{1+\delta_{\operatorname{limp}}^{2} /\left(\mathbf{B} \delta_{c}^{2}\right)} .
$$

Thus, $r_{1}$ tends to zero when the wave number ratio $\delta_{\text {limp }}^{2} / \delta_{c}^{2}$ tends to zero or when the term $\delta_{\operatorname{limp}}^{2} /\left(\mathbf{B} \delta_{c}^{2}\right)$ is much greater than 1 . The first condition is used as a parameter characterizing the influence of the frame, denoted the frame stiffness influence,

$$
\mathrm{FSI}=\frac{\delta_{\operatorname{limp}}^{2}}{\delta_{c}^{2}}=\frac{\widetilde{\rho}_{\text {limp }}}{\widetilde{\rho}_{c}} \frac{\hat{P}}{\widetilde{K}_{f}},
$$

with $\tilde{\rho}_{\text {limp }}, \widetilde{\rho}_{c}, \widetilde{K}_{f}$, and $\hat{P}$ given in Eqs. (33), (25), (11), and (10), respectively. As the Beranek criterion, ${ }^{7}$ the characteristic parameter FSI is expressed according to the ratio between the bulk moduli of the two phases. However, in the expression of FSI, each bulk modulus is divided by the density of their corresponding characteristic wave. Thus, Eq. (40) shows that FSI is frequency dependent and it decreases with the bulk modulus of the frame in vaccuo $\hat{P}$. Physically, it can be noticed that FSI is small when $\delta_{c}$ is great compared to $\delta_{\text {limp }}$, meaning that the interaction between the two associated waves is weak.

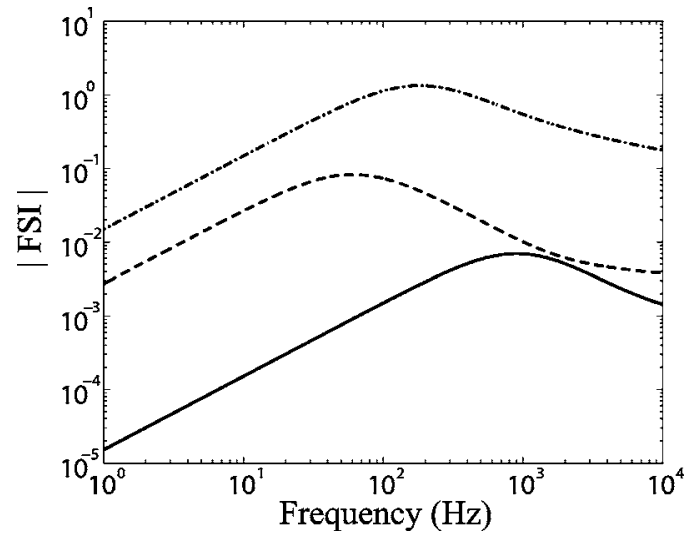

FIG. 3. Evolution of the parameter FSI as a function of the frequency: $(-)$ material A, (---) material B, (---) material C, (see Table I).

In the same way, using Eq. (22) and applying the firstorder Taylor series expansion of the square root function, the ratio $r_{2}$ can be expressed in terms of the parameter FSI as

$$
r_{2}=\mathbf{A}(1+\mathrm{FSI} / \mathbf{B})-\frac{\mathrm{FSI}}{1+\mathrm{FSI} / \mathbf{B}} .
$$

According to Eq. (41), when the parameter FSI tends to zero, $r_{2}$ tends to the value of the coefficient $\mathbf{A}$, which is close to 1 for all kinds of porous materials. Hence, the parameter FSI allows one to describe the asymptotic behavior of both $r_{1}$ and $r_{2}$ and thus the behavior of the ratio $\mu_{2} / \mu_{1}$. In the following, it is used to identify the porous materials according to the influence of their frame stiffness.

Figure 3 presents the FSI for three characteristic materials (see Sec. IV B and Table I). It is shown that the parameter FSI is frequency dependent and that the material $A$, which has the lower bulk modulus, has the smaller FSI.

\section{DESCRIPTION OF THE CONFIGURATIONS}

According to the previous analysis, the limp model can be applied instead of the Biot model if the parameter FSI tends to zero and if the amplitude of the frameborne wave is negligible compared to the amplitude of the airborne wave. This last condition depends primarily on the boundary conditions. In this paper, two different sets of boundary conditions representative of classical applications are investigated. For each configuration, the critical values of FSI under which the limp model can be used are determined from the

TABLE I. Measured properties of materials A, B, and C.

\begin{tabular}{lccc}
\hline \hline Porous & A & B & C \\
\hline Airflow resistivity: $\sigma\left(\mathrm{kN} \mathrm{s} / \mathrm{m}^{4}\right)$ & 105 & 23 & 57 \\
Porosity: $\phi$ & 0.95 & 0.95 & 0.97 \\
Tortuosity: $\alpha_{\infty}$ & 1 & 1 & 1.54 \\
Viscous length: $\Lambda(\mu \mathrm{m})$ & 35.1 & 54.1 & 24.6 \\
Thermal length: $\Lambda^{\prime}(\mu \mathrm{m})$ & 105.3 & 162.3 & 73.8 \\
Frame density: $\rho_{1}\left(\mathrm{~kg} / \mathrm{m}^{3}\right)$ & 17 & 58 & 46 \\
Young's modulus at $5 \mathrm{~Hz}: E(\mathrm{KPa})$ & 1.4 & 17 & 214 \\
Structural loss factor at $5 \mathrm{~Hz}: \eta$ & 0.1 & 0.1 & 0.115 \\
Poisson's ratio: $\nu$ & 0 & 0 & 0.3 \\
Beranek criterion at $5 \mathrm{~Hz}:\left|\tilde{K}_{f} / \hat{P}\right|$ & 70 & 6 & 0.4 \\
\hline \hline
\end{tabular}


response of a wide variety of porous materials. The following presents the configurations and the tested porous materials.

\section{A. The characteristic configurations}

Figure 1 presents the two configurations used to evaluate the difference between the Biot and the limp models. The chosen configurations are characteristic of the applications of the porous materials in industrial fields.

In configuration (a), the porous layer is attached to a rigid and motionless backing at $x=L$ and is hit by an acoustic plane wave at $x=0$. The normal-incidence absorption coefficient $\alpha$ of the porous material is derived from the complex reflection coefficient $R_{x}$ :

$$
\alpha=1-\left|R_{x}\right|^{2}, \quad R_{x}=\frac{Z_{n}-\rho_{f} \mathcal{C}_{f}}{Z_{n}+\rho_{f} \mathcal{C}_{f}},
$$

with $c_{f}$ the speed of sound in air and $Z_{n}$ the normal-incidence surface impedance. This impedance is given by the pressure to the normal velocity ratio in the free air close to the front face of the material; $Z_{n}=p(0) / v(0)$. Both pressure and velocity are determined using the appropriate boundary conditions: continuity of the normal stress in the solid and fluid phases with the external pressure at the fluid-porous interface $x=0$,

$$
\begin{aligned}
& \sigma_{x}^{s}(0)=-(1-\phi) p(0), \\
& \sigma_{x}^{f}(0)=-p(0),
\end{aligned}
$$

and continuity of the total flow

$$
j \omega\left[(1-\phi) u^{s}(0)+\phi u^{f}(0)\right]=v(0),
$$

with $u^{s}$ and $u^{f}$ given by Eqs. (27) and (28). On the surface of the porous layer in contact with the backing $(x=L)$, the displacement of the air and the frame are equal to zero,

$$
u^{s}(L)=u^{f}(L)=0 .
$$

An analytical formulation of $\alpha$ can be found in Ref. 2 (pp. $21,137)$. This coefficient is usually measured in an impedance tube ${ }^{15}$ and is used in building or automotive applications. ${ }^{16}$

In configuration (b), the porous layer is excited by a vibrating plate at $x=L$ and radiates in a infinite half-space at $x=0$. This configuration corresponds to trim panels, cars roofs, or airplane floors. The radiation efficiency factor $\sigma_{R},{ }^{17}$ defined as the ratio of the acoustic power radiated $\Pi_{a}$ over the vibratory power of the piston $\Pi_{v}$, is used as vibroacoustic response:

$$
\sigma_{R}=\frac{\Pi_{a}}{\Pi_{v}}=\frac{p(0) v^{*}(0)}{\rho_{f} c_{f} v_{w}^{2}} .
$$

A vibrating surface area of $1 \mathrm{~m}^{2}$ is considered here. Boundary conditions associated with this configuration are: ${ }^{18}$ continuity of stress and total flow at $x=0$ and Eqs. (43)-(45) are used. At $x=L$, the velocity of the fluid and the velocity of the frame are both equal to the wall velocity

$$
j \omega u^{s}(L)=j \omega u^{f}(L)=v_{w} .
$$

TABLE II. Range of values for the properties of the 256 simulated materials.

\begin{tabular}{lc}
\hline \hline Airflow resistivity: $\sigma\left(\mathrm{kN} \mathrm{s} / \mathrm{m}^{4}\right)$ & $1-100$ \\
Porosity: $\phi$ & 0.97 \\
Tortuosity: $\alpha_{\infty}$ & $1-2$ \\
Shape factor: $M$ & 1 \\
Viscous length: $\Lambda(\mu \mathrm{m})$ & $\sqrt{8 \alpha_{\infty} \mu^{\prime} / \sigma \phi M}$ \\
Thermal length: $\Lambda^{\prime}(\mu \mathrm{m})$ & $3 \Lambda$ \\
Frame density: $\rho_{1}\left(\mathrm{~kg} / \mathrm{m}^{3}\right)$ & $10-90$ \\
Young's modulus at $5 \mathrm{~Hz}: E(\mathrm{KPa})$ & $3-200$ \\
Structural loss factor at $5 \mathrm{~Hz}: \eta$ & $0.01-0.2$ \\
Poisson's ratio: $\nu$ & 0.3 \\
\hline \hline
\end{tabular}

For each configuration, the vibroacoustic response is derived using the transfer matrix method. ${ }^{2,19}$ This method assumes the multilayer has infinite lateral dimensions and uses a representation of plane wave propagation in different media in terms of transfer matrices. To ensure a onedimensional representation, the multilayer is excited by plane waves with normal incidence. The porous layer is either simulated using the Biot model presented in Sec. II B or the limp model presented in Sec. II C.

Note that the two characteristic configurations mainly differ by the kind of excitation applied to the frame: The frame will be less excited by an air domain than by a solid layer. Consequently, the limp model has a greater chance of being suitable for configuration (a) than for configuration (b).

\section{B. Material properties}

In order to study the behavior of a wide variety of porous materials, a set of 256 simulated materials and 3 real materials is used. The properties of the real materials presented in Table I have been measured at our laboratory. The viscoelastic characteristics of the porous frames, Youngs modulus E, and loss factor $\eta$, were measured at low frequency using the quasistatic method. ${ }^{20,21}$

Material A is a low density glass wool material with a very high airflow resistivity. This material is found in aerospace applications for thermal and sound insulation. Material $\mathrm{B}$ is a high density fibrous material and material $\mathrm{C}$ is a plastic foam with a stiff skeleton and a high airflow resistivity. Both materials $\mathrm{B}$ and $\mathrm{C}$ are found in automotive applications, for roof and floor treatment. According to the Beranek criterion (see last line in Table I), only the material A can be considered as a limp material $\left(\left|\widetilde{K}_{f} / \hat{P}\right|=70 \gg 20\right)$.

The 256 simulated materials are obtained by setting a random value for the main properties of a porous material: $E$ and $\eta$, respectively, the Young modulus and the loss factor of the frame in vacuum, $\rho_{1}$ the density of the porous material, $\sigma$ the airflow resistivity, and $\alpha_{\infty}$ the tortuosity. The range of values for the properties of the 256 porous materials are presented in Table II. The viscous characteristic length $\Lambda$ is derived from the shape factor given by Eq. (7). For most of the sound absorbing materials, the shape factor lies between 0.1 and 10 (Ref. 13) and it is chosen here equal to 1 . The thermal characteristic length $\Lambda^{\prime}$ is three times the viscous characteristic length $\Lambda$. For all the simulated materials, the 


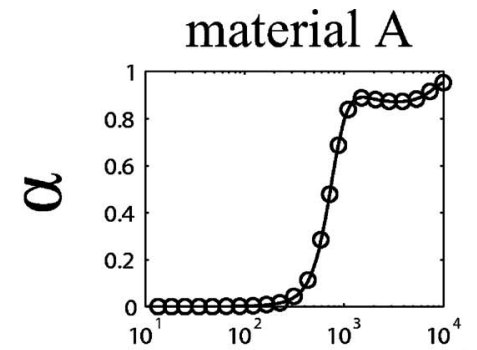

(a)

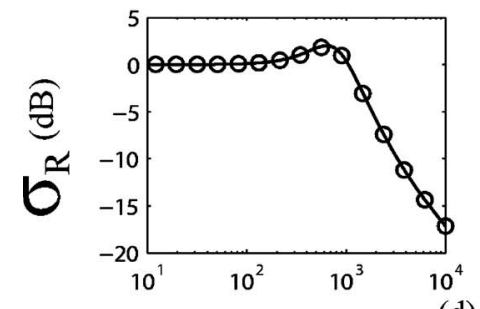

Frequency $(\mathrm{Hz})$

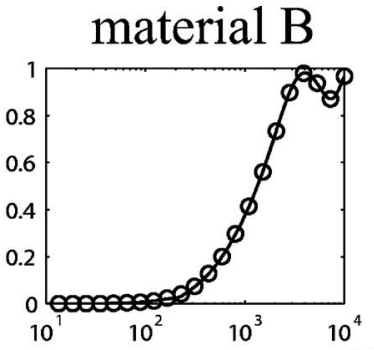

(b)

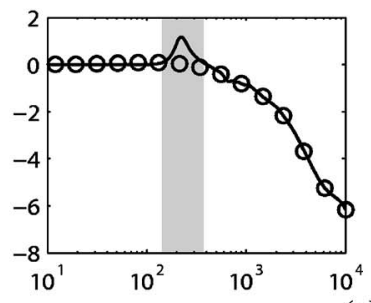

Frequency $(\mathrm{Hz})$

(e)

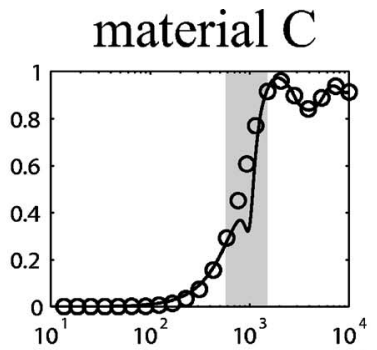

(c)

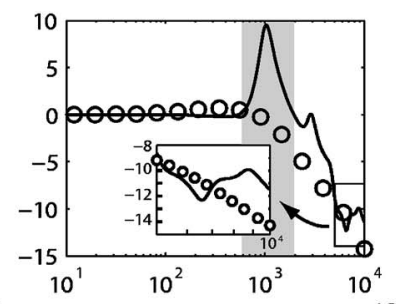

FIG. 4. Vibroacoustic response of the two configurations including the three real materials A, B, and C of thickness $2 \mathrm{~cm}$ : (first line) absorption coefficient $\alpha$, (second line) radiation efficiency $\sigma_{R}:(-)$ Biot model, $(\bigcirc)$ limp model. porosity is set to 0.97 and the Poisson ratio $\nu$ to 0.3 . The set of the randomly simulated materials is built to represent the variety of acoustical materials found in industrial applications.

\section{DETERMINATION OF CRITICAL FSI VALUES}

In Sec. III B the parameter FSI based on the physical properties of the material has been introduced. The next step is to identify, for each configuration, the critical values of FSI under which the limp model can be used instead of the Biot model. The method to determine a critical value is demonstrated in the case of the absorption coefficient of materials $\mathrm{A}, \mathrm{B}$, and $\mathrm{C}$ with a thickness of $2 \mathrm{~cm}$. Then, in order to get a critical value independent of the chosen material, the method is generalized with a set of 256 simulated materials, which properties are randomly determined among limits given in Table II. Results for the two configurations and thicknesses from 1 to $10 \mathrm{~cm}$ are finally presented.

\section{A. Analysis on materials A, B, and C}

\section{Three characteristic frequency ranges}

Because the influence of the frame is frequency dependent, the study of the critical values of FSI will be carried out in three characteristic frequency bands centered around the first $\lambda / 4$ resonance frequency of the frameborne wave, $f_{r}$. This frequency can be estimated by simply considering the properties of the frame in vacuo: ${ }^{22}$

$$
f_{r} \approx \frac{1}{4 L} \sqrt{\frac{\hat{P}}{\rho_{1}}}=\frac{1}{4 L} \sqrt{\frac{E \frac{(1-\nu)}{(1+\nu)(1-2 \nu)}}{\rho_{1}}},
$$

where $L$ is the thickness of the porous layer. In the vicinity of $f_{r}$, the frame stiffness can have a great influence on both the absorption coefficient ${ }^{16,22}$ and the radiation efficiency. ${ }^{18}$

This is illustrated in Fig. 4 (gray zones) where the Biot and the limp simulations of both configurations are presented for materials $\mathrm{A}, \mathrm{B}$, and $\mathrm{C}$ of thickness $2 \mathrm{~cm}$. In the case of the absorption configuration, a decrease of the absorption coefficient is observed at the frequency $f_{r}$ around $1000 \mathrm{~Hz}$ for material $\mathrm{C}$ [see Fig. 4(c)]. In the case of the radiation configuration, an increase of the radiation efficiency is observed around $200 \mathrm{~Hz}$ for material B [see Fig. 4(e)] and $1000 \mathrm{~Hz}$ for material C [see Fig. 4(f)]. Higher orders modes $(3 \lambda / 4, \ldots)$ also have an influence in the case of the radiation of material C [Fig. 4(f)].

In the following, three characteristic zones are chosen according to the frequency $f_{r}$ [Eq. (49)]: $\left[1, f_{r} / 2\right]$, the low frequency range $(\mathrm{LF}) ;\left[f_{r} / 2,2 f_{r}\right]$, the midfrequency range $(\mathrm{MF})$; and $\left[2 f_{r}, 10^{4}\right]$, the high frequency range (HF).

\section{Estimation of the difference between the models}

The difference between the limp and the Biot models is given in third octave bands by the absolute value of the difference between the two vibroacoustic responses $V$ ( $V$ being either $\alpha$ or $\left.\sigma_{R}\right): \Delta V=\left|V_{\text {Biot }}-V_{\text {Limp }}\right|$. The frequency band $1-10000 \mathrm{~Hz}$ is divided in 41 third octave bands. The maximum accepted difference between the two models is set to 0.1 for the absorption coefficient and to $3 \mathrm{~dB}$ for the radiation efficiency. These values correspond to a classical industrial demand.

\section{Method to determine the FSI critical values}

The objective of this section is to determine, according to the configuration and the frequency domain, the maximum value of the FSI under which the limp model can be used. This maximum value is called "critical value."

The method to obtain a critical value is presented for the absorption configuration with materials $\mathrm{A}, \mathrm{B}$, and $\mathrm{C}$ having a thickness of $2 \mathrm{~cm}$. The difference between the two simulations $\left|\alpha_{\text {Biot }}-\alpha_{\text {Limp }}\right|$ is presented in Fig. 5 as a function of FSI in the three frequency ranges for each material. The higher value of FSI above which the maximum acceptable difference between the two models (here 0.1) is exceeded, is found in the medium frequency range at $\mathrm{FSI}=0.5$ [vertical dotted line on Fig. 5(b)]: This value is set as critical value. No critical values are found in the low and the high frequency ranges. 


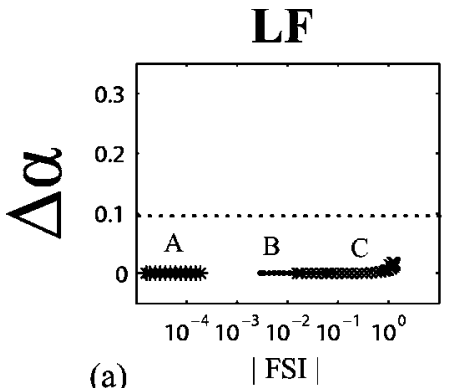

(a)

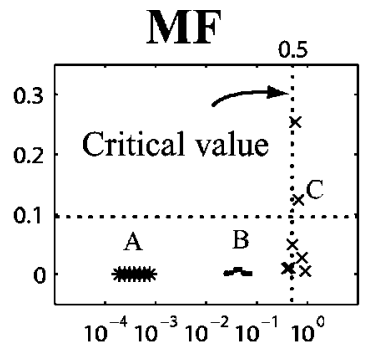

(b)

\section{B. Generalization using 256 simulated porous materials}

To establish critical values of FSI that are independent of the chosen material, the preceding study is generalized by using the 256 simulated materials (see Table II). Figures 6(a)-6(c) are equivalent to Fig. 5, but present the difference between the limp and the Biot absorption coefficient derived for 256 simulated materials having a thickness of $2 \mathrm{~cm}$. Figures 6(d)-6(f) present the same results in the case of the radiation configuration.

The first noticeable tendency is that the value of the difference between the limp and the Biot models increases when the value of FSI increases. This is in agreement with the parameter behavior, which increases with the bulk modulus of the frame (see Sec. III B). As stated previously, the critical value of FSI is set when the first parametrized curve exceeds the maximum accepted difference. However, this method can be inappropriate in this case because the limit can depend slightly on the random set of simulated materials. Thus, a simple statistic indicator called the "confidence rate" is used to set the critical values. The FSI range is first divided into 10 bands from $10^{-4}$ to $10^{0}$ in a $\log$ scale. In each FSI band, the confidence rate gives the number of points below the acceptable limit $(0.1$ or $3 \mathrm{~dB})$ over the number of points present in this FSI range. This rate, given in percent (solid gray line in Fig. 6), indicates the reliability of using the limp model according to the value of FSI.

The confidence rate of $95 \%$ is chosen as a threshold to

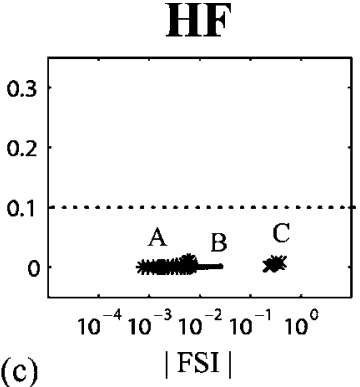

FIG. 5. Difference between the Biot and limp models on the absorption coefficient $\alpha$ of 2 -cm-thick materials A $(*)$, B $(\bigcirc)$, and $\mathrm{C}(\times)$, functions of their FSI parameter in the three frequency domains: (a) low frequency band, (b) medium frequency band, and (c) high frequency band.

set the critical value of FSI, called FSI $_{95}$. For a FSI larger than $\mathrm{FSI}_{95}$, it is considered that the vibroacoustic response may become notably sensitive to the frame stiffness for most of the porous materials and the Biot model should be preferred. Note that the number of FSI bands used to derive the confidence rate is sufficiently important so that the critical value $\mathrm{FSI}_{95}$ does not depend on this number.

In the case of the absorption coefficient in the medium frequency range [see Fig. 6(b)], the critical value is $\mathrm{FSI}_{95}$ $=0.16$. No critical values are set in the low and high frequency ranges: The limp model can be used to predict the absorption coefficient for all the acoustic materials. These results obtained for a thickness of $2 \mathrm{~cm}$ are summarized in an abacus presented in Fig. 7(a). In this figure, a white cell corresponds to the FSI values for which the limp model can be used. A gray cell corresponds to the FSI values for which the Biot model should be preferred.

Let us consider now the radiation configuration. Figures 6(d)-6(f) present the difference between the two models as a function of FSI, and the obtained critical FSI are summarized in Fig. 7(b). As shown Fig. 6(d), no critical FSI values are set in the low frequency range: The radiation efficiency of a covered plate can be predicted using the limp model for all the acoustic materials for frequencies at less than two times below the first resonance frequency of the frame $f_{r}$. Critical values of FSI are set in the medium and the high frequency ranges as seen in Figs. 6(e) and 6(f). The difference between the Biot and the limp models can be great in the high fre-

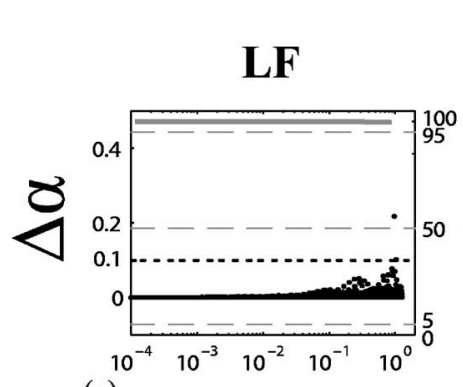

(a)

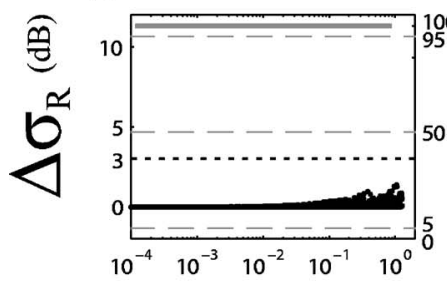

(d)

| FSI |

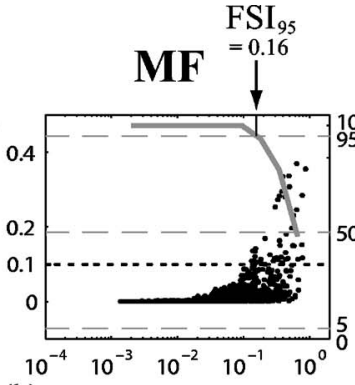

(b)

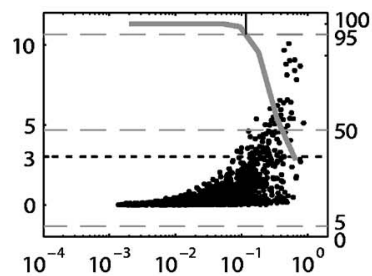

(e)

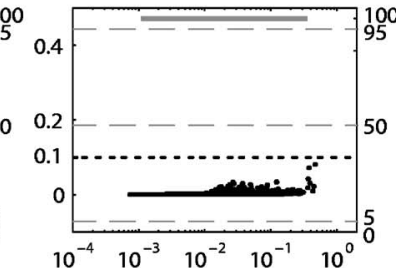

(c)$$
\begin{aligned}
& 10 \\
& 5 \\
& 3 \\
& 0 \\
& 10
\end{aligned}
$$

(f)
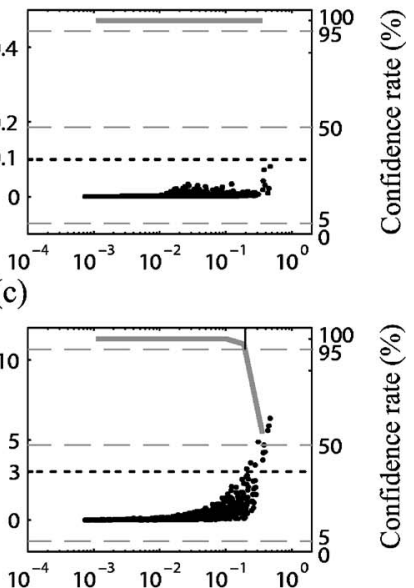

| FSI $\mid$
FIG. 6. Evolution of the difference between the Biot and limp models as a function of FSI for the two configurations including the 256 simulated materials of thickness $2 \mathrm{~cm}$ : (a)-(c) absorption coefficient, (d)-(f) radiation efficiency of a covered plate; in the three frequency ranges: (first column) low frequency band LF, (second column) medium frequency band MF, and (third column) high frequency band HF. 

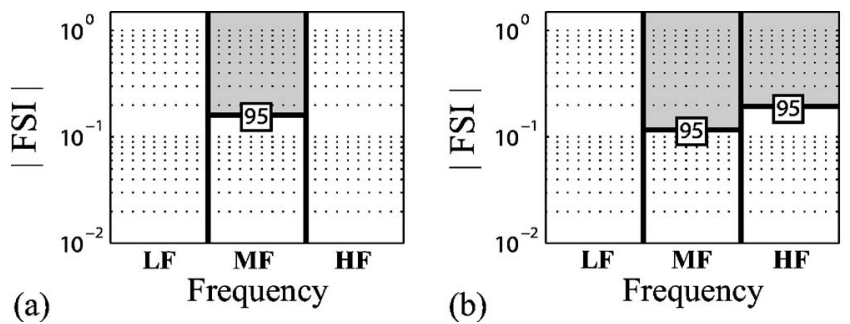

FIG. 7. Critical values of FSI in the three frequency ranges for the two configurations including a porous layer of thickness $2 \mathrm{~cm}$ : (a) absorption coefficient, (b) radiation efficiency of a covered plate; white area: The FSI is below the critical value $\mathrm{FSI}_{95}$, light gray area: The FSI is above the critical value $\mathrm{FSI}_{95}$.

quency range because higher order resonances $(3 \lambda / 4,5 \lambda / 4, \ldots)$ can be excited in the frame thickness and have a noticeable influence on the radiation efficiency.

\section{Critical values as a function of the thickness}

The study of the critical values of FSI is carried out for thicknesses from 1 to $10 \mathrm{~cm}$. Figure 8 presents, in the three frequency domains, the evolution of the critical values of FSI as a function of the layer thickness for both configurations.

In the low frequency range [Figs. 8(a) and 8(d)], most of the configurations do not exhibit a critical FSI. In this frequency range, the wavelength of the frameborne wave is large compared to the thickness of the layer and thus the frame thickness tends to be constant.

In the medium frequency range [Figs. 8(b) and 8(e)], centered around the quarter wavelength resonance, it is shown that the critical values of FSI slightly decrease when the porous thickness increases. This tendency is more pronounced in the high frequency range as shown Figs. 8(c) and $8(f)$. For example, the absorption coefficient of a material having a FSI of $10^{\circ}$ can be simulated with the limp model only for thickness up to $4 \mathrm{~cm}$ [see Fig. 8(c)]. This shows that the difference between the Biot and the limp models increases with the thickness.

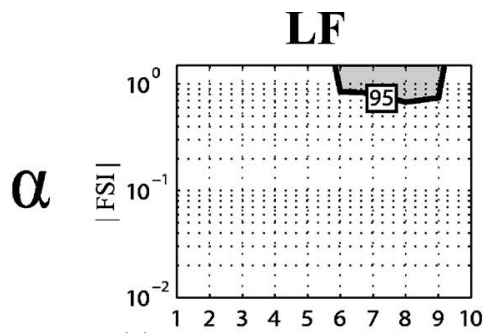

(a)

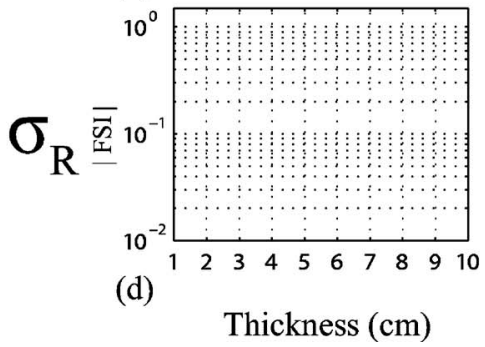

(b)
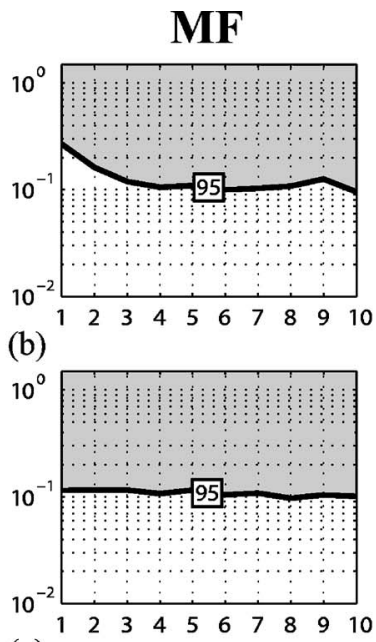

(e)

Thickness (cm)

\section{DISCUSSION}

It is possible to explain how the FSI criterion should be used. The prediction of the frequency bands for which the limp model can be used is proposed in the case of the absorption coefficient of materials A, B, and C (see Table I) of thickness $2 \mathrm{~cm}$. This method only involves the calculation of the FSI parameter [Eq. (40)] of the tested material and avoids solution of the absorption or radiation problems using the full Biot model and limp model (see Sec. IV A). Finally, the FSI criterion is compared to the Beranek criterion and, in the particular case of the absorption configuration, with classical "rigid frame" criteria.

\section{A. The evaluation procedure and application}

The procedure for determining the frequency ranges in which the limp model is valid is as follows:

(i) The properties of the porous materials have to be measured (see Table I for materials A, B, and C),

(ii) The FSI is derived using Eq. (40); the various parameters $\left(\hat{P}, \tilde{\rho}_{c}\right.$, and $\left.\tilde{\rho}_{\text {limp }}\right)$ are evaluated using Eqs. (10), (25), and (33); FSI curves are drawn for materials A, B, and C in Figs. 9(a)-9(c), respectively.

(iii) The frequency bands characteristic of the porous behavior are estimated from the center frequency of the medium frequency band $f_{r}$ [Eq. (49)]; for a 2-cm-thick layer, the frame resonance is $f_{r \mathrm{~A}}$ $\approx 113 \mathrm{~Hz}$ for material $\mathrm{A}, f_{r \mathrm{~B}} \approx 214 \mathrm{~Hz}$ for material $\mathrm{B}$, and $f_{r \mathrm{C}} \approx 990 \mathrm{~Hz}$ for material $\mathrm{C}$.

(iii) The critical values of FSI are chosen in Fig. 8 according to the studied configuration, the thickness of the porous layer, and the frequency band. The data corresponding to the absorption configuration with a layer having a thickness of $2 \mathrm{~cm}$ are added on the FSI curves presented in Figs. 9(a)-9(c).

The comparison between the FSI curve and the critical value $\mathrm{FSI}_{95}$ enables identification of the frequency bands where the limp model can be used. The results of the comparison for the two configurations are summarized in Figs.

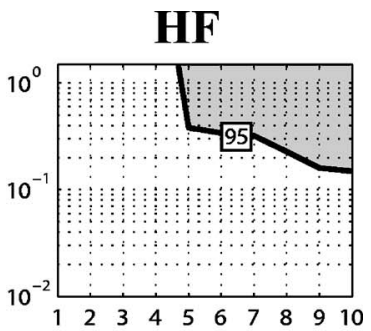

(c)

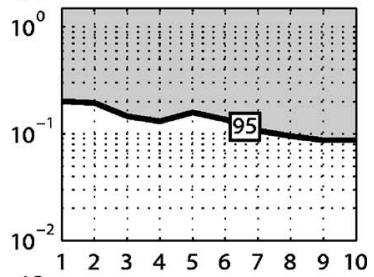

(f)
Thickness (cm)
FIG. 8. Evolution of the FSI critical values as function of the thickness for the two configurations [(a)-(c) absorption coefficient, $((\mathrm{d})-(\mathrm{f})$ radiation efficiency] in the three frequency ranges: (a), (d) low frequency band LF, (b), (e) medium frequency band MF, (c), (f) high frequency band HF. White zone: limp model valid; grayed zone: limp model not valid. 


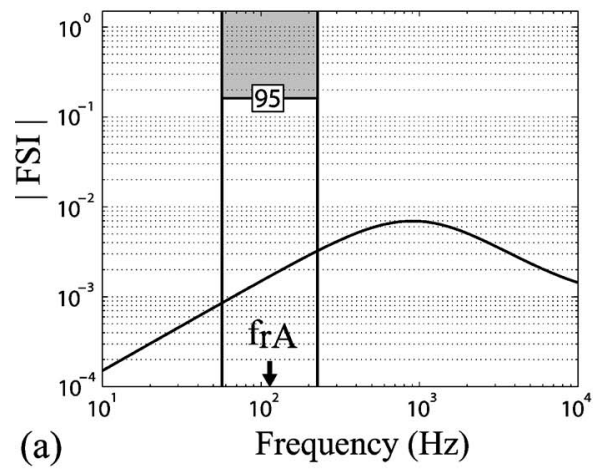

(a)

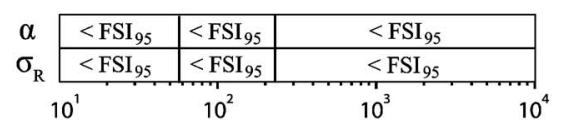

(d)

Frequency $(\mathrm{Hz})$

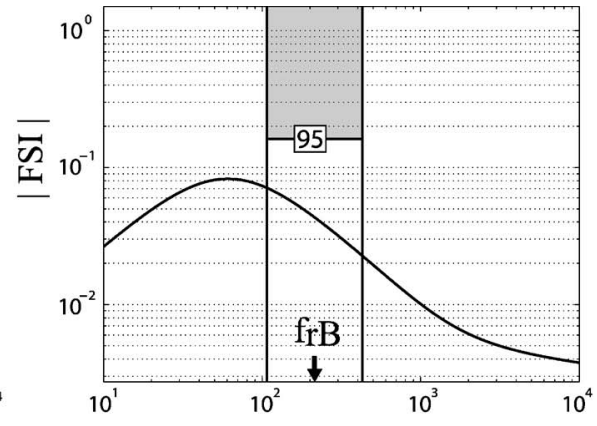

(b)

Frequency $(\mathrm{Hz})$

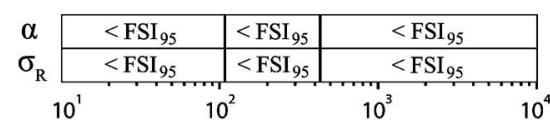

(e)

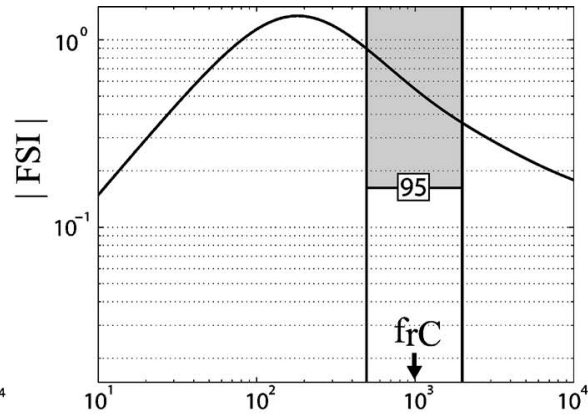

(c)

Frequency $(\mathrm{Hz})$

(f)

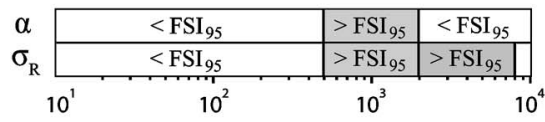

Frequency $(\mathrm{Hz})$

FIG. 9. (a)-(c) FSI curves and critical values obtained in the case the absorption configuration for a porous layer 2 cm thick: (a) material A, (b) material B, (c) material C; (d)-(f) FSI compared to the critical values for the two characteristic configurations in the three frequency domains: (d) material A, (e) material $\mathrm{B}$, and (f) material C.

9(d)-9(f). It is shown that materials A and B can be simulated using the limp model for both the absorption and the radiation configurations over the whole frequency range $\left(\mathrm{FSI}<\mathrm{FSI}_{95}\right)$. These predictions agree with the simulations presented in Figs. 4(a), 4(b), 4(d), and 4(e). Note that the increase of the radiation efficiency of material B [see Fig. $4(\mathrm{e})]$ induced by the frame resonance does not exceed the maximum accepted difference of $3 \mathrm{~dB}$. For material $\mathrm{C}$, it is predicted that the Biot model should be used in the medium frequency range for both configurations $\left(\mathrm{FSI}>\mathrm{FSI}_{95}\right)$. This is confirmed in Figs. 4(c) and 4(f). For the radiation configuration, the use of the Biot model is recommended up to $8 \mathrm{kHz}$ [see Fig. 9(f)]. For higher frequencies, the limp model can be used. This prediction is validated in Fig. 4(f) where the difference between the two simulations is great around the $3 \lambda / 4$ frame resonance (from 2 to $5 \mathrm{kHz}$ ) and does not exceed $3 \mathrm{~dB}$ above $8 \mathrm{kHz}$.

\section{B. Comparison with other criteria}

According to the Beranek criterion, ${ }^{7}$ only the material A can be simulated with the limp model (for material A, $\widetilde{K}_{f} / \hat{P}=70 \gg 20$, see Table I). The FSI prediction for this material agrees with Beranek prediction: The absorption coefficient and the radiation efficiency can be simulated with the limp model in the whole frequency range. For the two other materials, the criterion proposed by Beranek is too restrictive: The use of the limp model is banned for both materials B and C $\left(\widetilde{K}_{f} / \hat{P}=6\right.$ for material B and 0.4 for material C).

In the particular case of the absorption configuration, the material is generally modeled using the rigid frame assumption (see Sec. II C). To ensure the validity of this assumption, one can use a frequency criterion proposed by Biot. ${ }^{1}$ This criterion gives a high critical frequency limit above which the viscous forces only have a noticeable effect in a layer of air in contact with the frame, of equal thickness to the viscous skin depth: The frame cannot be excited by an acoustical wave and can be considered rigid. This frequency is expressed as the ratio of the airflow resistivity of the porous material over the density of the interstitial fluid: $f_{\text {Biot }}$ $=\sigma / 2 \pi \rho_{f}$. Applied to materials A, B, and C, this criterion allows the use of the rigid frame model above $13881 \mathrm{~Hz}$ for material A, $3041 \mathrm{~Hz}$ for material B, and $7535 \mathrm{~Hz}$ for material C. As stated previously, the parameter FSI allows the use of the limp model in the whole frequency range for both fibrous materials $\mathrm{A}$ and $\mathrm{B}$, and recommends the use of the Biot model in the vicinity of the frame resonance for the material C. Hence, in this case, the frame can be excited by the motion of the air (predictable with the criterion $f_{\text {Biot }}$ ) but, since the stiffness has no influence (predictable with FSI), the "equivalent fluid" limp model can nevertheless be used.

Pilon et al. ${ }^{16}$ proposed a criterion to study the influence of the mounting conditions on the measurement of the sound absorption coefficient in a duct. This criterion, called FAE and defined as $\mathrm{FAE}=\sigma E / \rho_{1}^{2}$, gives informations about the influence of the frame around its frequency resonance $f_{r}$. For material $\mathrm{C}$, the $\mathrm{FAE}$ is equal to $5.7 \mathrm{MW} / \mathrm{kg}$ and greatly superior to the critical value $(1 \mathrm{MW} / \mathrm{kg})$, which indicates that the acoustic behavior of the foam is very sensitive to the frame vibration in the vicinity of $f_{r}$. The prediction is in good agreement with the FSI criterion.

\section{CONCLUSION}

In this paper, a criterion identifying which porous materials can be modeled with the one-wave limp model instead of the poroelastic Biot model has been investigated. The identification process relies on a new parameter derived from the properties of the material: the frame stiffness influence. This parameter, based on the compressional wave numbers, expresses the influence of the frameborne wave on the fluid phase displacement. The method consists in evaluating the FSI for a given material and comparing it with critical values determined for two characteristic configurations: absorption of a porous layer bonded on a rigid backing and radiation of a vibrating plate covered by a porous layer. It is shown that the frequency bands predicted to be simulated with the limp or the Biot model using this method are in close agreement 
with one-dimensional simulations. Even if the application cases are one dimensional, they involve both Biot compressional waves. It seems realistic to apply this criterion to three-dimensional cases where the shear wave is not mainly excited, which is the case for a wide variety of industrial applications. Compared with other criteria, the FSI criterion provides a more accurate information in terms of frequency and confirms that the limp model is less restrictive than the rigid frame one.

\section{ACKNOWLEDGMENTS}

The authors would like to thank the CNRS and Région Pays de la Loire for their financial support.

${ }^{1}$ M. A. Biot, "The theory of propagation of elastic waves in a fluidsaturated porous solid. I. Low frequency range. II. Higher frequency range," J. Acoust. Soc. Am. 28, 168-191 (1956).

${ }^{2}$ J. F. Allard, Propagation of Sound in Porous Media: Modelling Sound Absorbing Materials (Elsevier Applied Science, London, 1993).

${ }^{3}$ R. Panneton and N. Atalla, "An efficient scheme for solving the threedimensional poroelasticity problem in acoustics," J. Acoust. Soc. Am. 101, 3287-3298 (1998).

${ }^{4}$ N. E. Hörlin, M. Nordström, and P. Göransson, "A 3-D hierarchical FE formulation of Biots equations for elastoacoustic modeling of porous media," J. Sound Vib. 254, 633-652 (2001).

${ }^{5}$ N. Atalla, R. Panneton, and P. Debergue, "A mixed displacement-pressure formulation for poroelastic materials," J. Acoust. Soc. Am. 104, 14441452 (1998).

${ }^{6}$ S. Rigobert, N. Atalla, and F. Sgard, "Investigation of the convergence of the mixed displacement pressure formulation for three-dimensional poroelastic materials using hierarchical elements," J. Acoust. Soc. Am. 114, 2607-2617 (2003).

${ }^{7}$ L. L. Beranek, "Acoustical properties of homogeneous, isotropic rigid tiles and flexible blankets," J. Acoust. Soc. Am. 19, 556-568 (1947).

${ }^{8}$ K. U. Ingard, Notes on Sound Absorption Technology (Noise Control
Foundation, New York, 1994).

${ }^{9}$ O. Dazel, B. Brouard, C. Depollier, and S. Griffiths, "An alternative Biots displacement formulation for porous materials," J. Acoust. Soc. Am. 121, 3509-3516 (2007).

${ }^{10}$ C. Zwikker and C. W. Kosten, Sound Absorption Materials (Elsevier Applied Science, New York, 1949).

${ }^{11} \mathrm{P}$. Göransson, "A weighted residual formulation of the acoustic wave propagation through a flexible porous material and a comparison with a limp material model," J. Sound Vib. 182, 479-494 (1995).

${ }^{12} \mathrm{P}$. Göransson, "Acoustic finite element formulation of a flexible porous material: A correction for inertial effects," J. Sound Vib. 185, 559-580 (1995).

${ }^{13}$ D. L. Johnson, J. Koplik, and R. Dashen, "Theory of dynamic permeability and tortuosity in fluid-saturated porous media," J. Fluid Mech. 176, 379-402 (1987).

${ }^{14}$ Y. Champoux and J. F. Allard, "Dynamic tortuosity and bulk modulus in air-saturated porous media," J. Appl. Phys. 70, 1975-1979 (1991).

${ }^{15}$ A. F. Seybert and D. F. Ross, "Experimental determination of acoustic properties using a two-microphone random-excitation technique," J. Acoust. Soc. Am. 61, 1362-1370 (1977).

${ }^{16}$ D. Pilon, R. Panneton, and F. Sgard, "Behavioral criterion quantifying the edge-constrained effects on foams in the standing wave tube," J. Acoust. Soc. Am. 114, 1980-1987 (2003).

${ }^{17}$ C. E. Wallace, "Radiation resistance of a rectangular panel," J. Acoust. Soc. Am. 51, 946-952 (1972).

${ }^{18}$ O. Doutres, N. Dauchez, and J. M. Génevaux, "Porous layer impedance applied to a moving wall: Application to the radiation of a covered piston," J. Acoust. Soc. Am. 121, 206-213 (2007).

${ }^{19}$ B. Brouard, D. Lafarge, and J. F. Allard, "A general method of modeling sound propagation in layered media," J. Sound Vib. 183, 129-142 (1995).

${ }^{20}$ N. Dauchez, M. Etchessahar, and S. Sahraoui, "On measurement of mechanical properties of sound absorbing materials," Second Biot Conference on Poromechanics, Grenoble, 2002.

${ }^{21}$ M. Etchessahar, S. Sahraoui, L. Benyahia, and J. F. Tassin, "Frequency dependence of elastic properties of acoustic foams," J. Acoust. Soc. Am. 117, 1114-1121 (2005).

${ }^{22}$ J. F. Allard, C. Depollier, P. Guignouard, and P. Rebillard, "Effects of a resonance of the frame on the surface impedance of glass wool of high density and stiffness," J. Acoust. Soc. Am. 89, 999-1001 (1991). 